\title{
Chapter 7 \\ Development of a 3" LaBr3 SiPM-Based \\ Detection Module for High Resolution \\ Gamma Ray Spectroscopy and Imaging
}

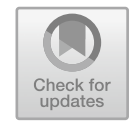

\author{
Giovanni Ludovico Montagnani
}

\subsection{Introduction}

My thesis work entails the design of each element of the instrument (Fig. 7.1). Starting from the SiPM tile and the optimization of the instrument mechanics, through to the development of the electronics boards and custom ASICs. The Gain Amplitude Modulated Multichannel ASIC (GAMMA) was developed in order to match the project requirements for a large charge dynamic range and full scale range. The ASIC is meant to cope with the $14 \mathrm{~dB}$ charge range provided by the SIPMs and to provide this information minimizing the statistical contribution to resolution degradation. The exploitation of a Gated Integrator filter with self triggering capabilities was meant to optimize the signal collection versus the integration of SiPM dark current. During the years of work, me and my team of students, developed a first 8-channel ASIC, and a second release exploiting 16 analog channels and multiplexers. The ASIC main schematic is represented by Fig. 7.2: the core of the chip is the 16 channels stack which exploits a current input stage, the Gated Integrator with Track and Hold feature, an Active Gain Control and a Baseline Holder circuit [2].

\subsection{Development}

A peculiar feature of the GAMMA ASIC is the Active Gain Change mechanism, that profit from a "predictive" analog algorithm to choose the best gain of the amplifier to process the input pulse in order to minimise the contribution of the output noise as depicted by Fig. 7.3. Another important feature of the analog filter is the Baseline

\footnotetext{
G. L. Montagnani ( $\varangle)$

Dipartimento di Elettronica, Informazione e Bioingegneria, Politecnico di Milano, Piazza Leonardo da Vinci 32, Milano, Italy

e-mail: giovanni.montagnani@polimi.it

(C) The Author(s) 2021

A. Geraci (ed.), Special Topics in Information Technology,

PoliMI SpringerBriefs, https://doi.org/10.1007/978-3-030-62476-7_7
} 
Fig. 7.1 Developed

spectrometer enclosure. The cylindric shape is designed to contain the crystal, the hexagonal flange allows the exploitation of the system in laboratory setups and the upper box hosts the electronics

Fig. 7.2 GAMMA ASIC main schematic. The analog channel exploits a current input stage, the Gated Integrator with Track and Hold feature, an Active Gain Control and a Baseline Holder circuit
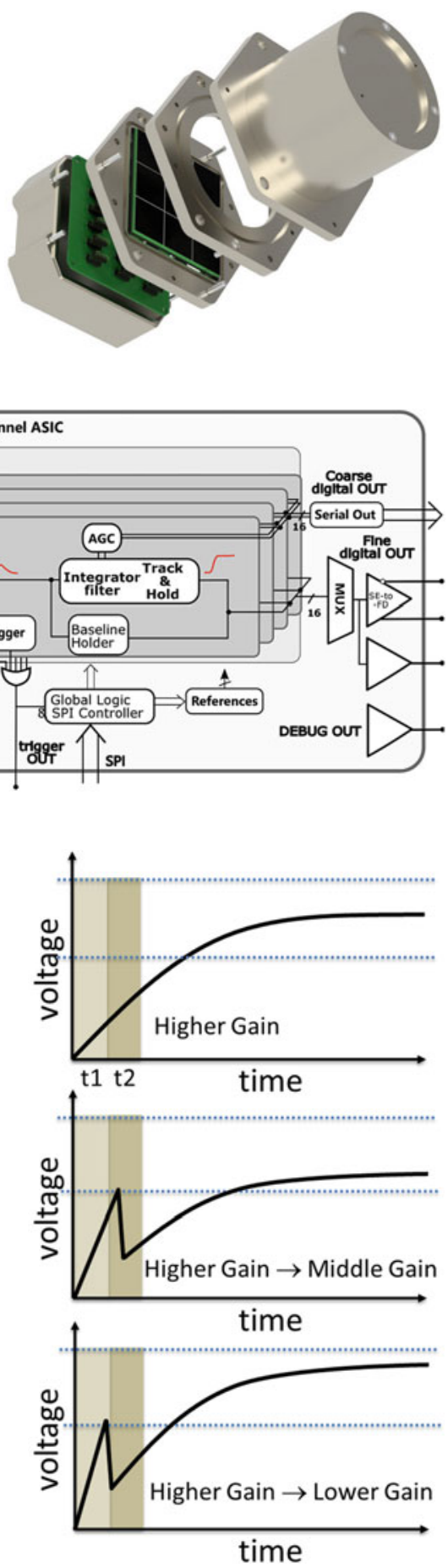

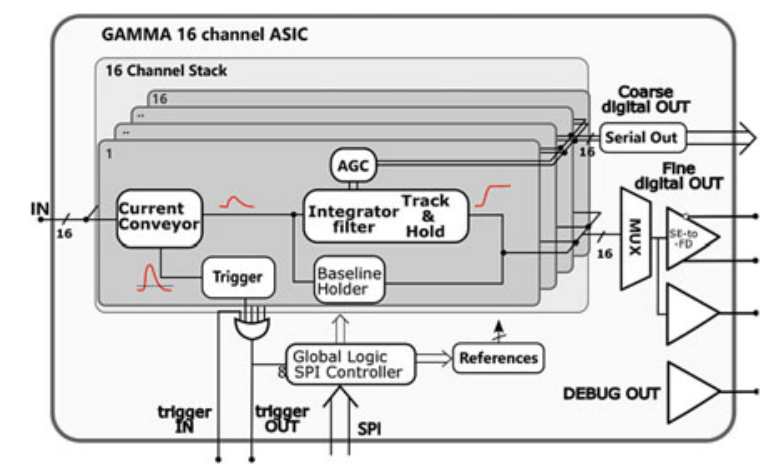

Fig. 7.3 Active Gain Change time diagram. The ASIC swaps the integrating capacitance during the integration time sensing the output voltage 

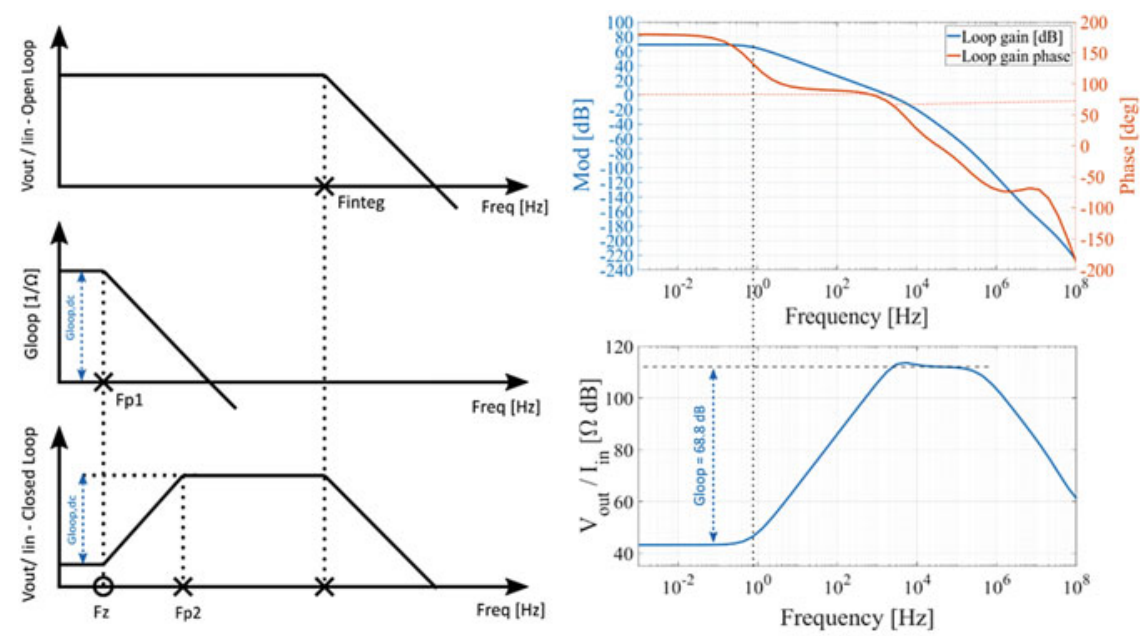

Fig. 7.4 Baseline Holder filter working principle. By placing a very low frequency low pass filter in feedback to the Gated Integrator a bandpass effect is obtained

Holder used to avoid DC current integration. This $1 \mathrm{~Hz}$ low pass filter with non-linear behaviour is used in feedback to the Gated Integrator filter, in order to drain the DC current from the input stage as depicted by Fig. 7.4. The non-linearity is useful to avoid a dependence of the subtracted DC current from the amplitude of the current pulses, but it must be dimensioned carefully in order to avoid unwanted instability issues [1]. The final 16 channel ASIC also hosts an SPI programmable memory to tune the filters parameters trough 7 internal DACs and a differential output buffer to match the requirements of the external 14 differential ADC. The ASIC and the ADC are placed on a small PCB module to be placed on a larger motherboard for improved connectivity and modularity [3].

Data acquisition and the biasing of the detectors were also designed in order to simplify the use of the instrument in operative conditions. An important feature that allowed state of the art results is the SiPM gain stabilization through overvoltage correction versus temperature variation. An ARTIX-7 FPGA, handles the acquisition from the ASICs, data storage, processing, reconstruction algorithm and USB 2.0 communication [5] (Fig. 7.5).

Experimental measurements were performed in intermediate development steps, confirming the high performance of the developed instrument. Considering that the main motivation of this work was to develop the first instrument capable of detecting rays from hundreds of thousands of electronvolts to tens of millions of electronvolts with high efficiency and state of the art energy resolution, the large number of active channels (120) was mandatory to achieve both spatial sensitivity and energy resolution. The measurements performed on a preliminary detection module have demonstrated the ability of the system to consistently achieve results in line with expectations-even better in some cases-within the restrictions given by the lim- 
Fig. 7.5 SiPM readout and biasing circuitry. The system hosts 9 GAMMA ASICs, $\mathrm{DC} / \mathrm{DC}$ regulators and FPGA for post-processing

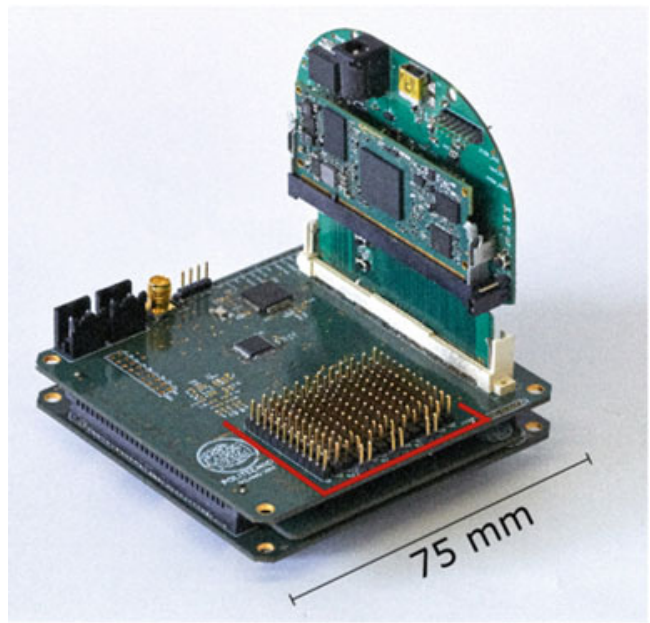

ited number of readout channels and by the simplified microcontroller-based DAQ. A record energy resolution of $2.58 \%$ FWHM at $662 \mathrm{keV}$ [4] has been achieved coupling the designed detector with a co-doped $\mathrm{LaBr} 3$ crystal by Saint-Gobain as reported in Figs. 7.6 and 7.7. This is, to the author's knowledge, the best resolution obtained coupling SiPM with scintillator crystals. A spatial resolution better than $10 \mathrm{~mm}$ at the center of the matrix has been obtained dividing the matrix in just 8 macro-pixels. The measurement dataset, shown in Fig. 7.8, was used to train an algorithm capable of reconstructing the horizontal position of interaction with sufficient precision. The next step of the project is to complete the 144-SiPM matrix readout system coupling each SiPM with a dedicated ASIC input: this is achieved by exploiting a total of 9 16-channels ASIC, of which a first release has been tested in parallel to this thesis and the results are reported in the dedicated chapter. The writing of this thesis occurred during the beginning of the testing phase. However, up to now, the only spectroscopic results available were obtained with single 8-channel or 16-channel ASICs in a reduced energy range. It will be possible to read individually the 120 SiPM of the matrix, increasing the energy full scale range to meet the project specification of $20 \mathrm{MeV}$. The combination of the preliminary system developed during this thesis work with the new 16 channels ASICs and the FPGA-based acquisition system will allow to obtain the ultimate performances targeted by the GAMMA project, satisfying all the requirements of the application: 120 non-merged SiPMs will allow to reach the target energy resolution while reaching the full required dynamic range, in conjunction with excellent spatial resolutions thanks to a large number of small pixels and to the promising neural-network-driven algorithm whose operation will be further refined. The new data acquisition system will permit to acquire data from a all the ASICs even at high event rates, together with an easy management of the SiPM bias voltage to compensate for gain variation due to temperature.

Future developments of my work could involve a better exploitation of the FPGA capabability other than mere improvements of the developed hardware and 
Fig. 7.6 Spectra comparison between the 8 channel and the 16 channel ASIC readout of the same 3" diameter $\mathrm{LaBr} 3$ crystal

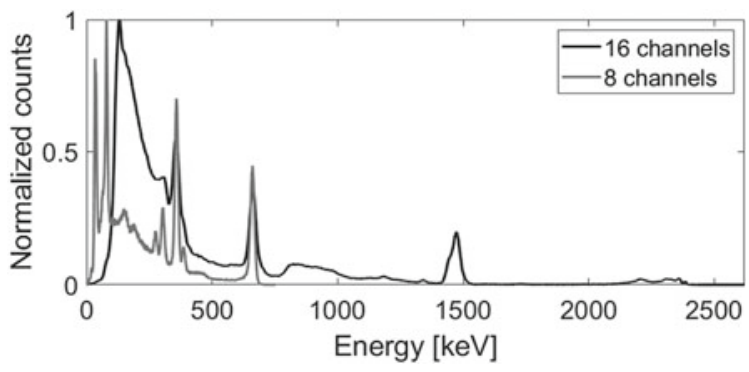

Fig. 7.7 Acquired spectrum with record resolution of 2.58\%@66keV obtained from the readout of a 3" diameter $\mathrm{LaBr} 3 \mathrm{Sr}$ codoped crystal

Fig. 7.8 Training dataset used for position reconstruction algorithm. Average signal distribution (vertical) for the 8 different positions corresponding to the centers (horizontal) of the pixels and interaction point reconstruction results using a cross-correlation-based algorithm
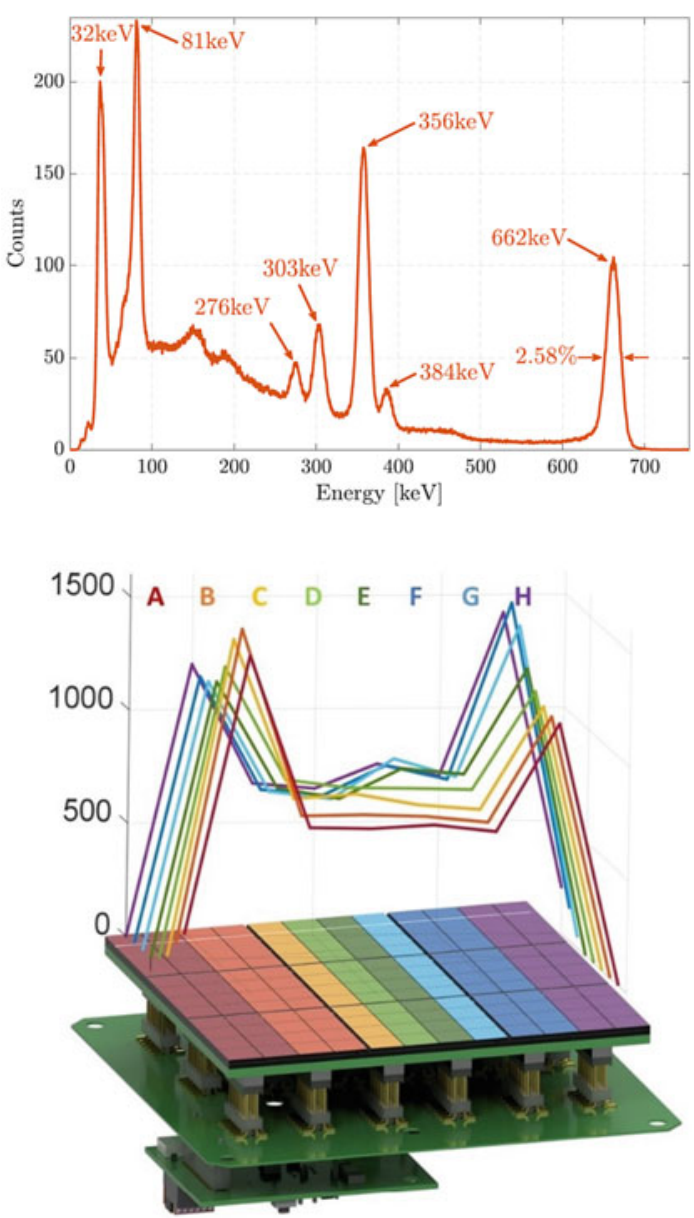

bug corrections. Availability of computationally efficient Machine Learning (ML) algorithms, combined to high performance, easily configurable FPGA and microcontrollers can push the state-of-the-art performances of modern embedded systems towards better results using new, distributed architectures. The sharing of com- 
putational complexity in different node of a network or electronic system when implementing ML poses interesting challenges and the need for new architecture paradigms. The high energy resolution gamma spectroscopy presented in this thesis work could use K-Nearest Neighbour algorithms for interaction point reconstruction in thick scintillation crystals coupled to large arrays of Silicon Photomultipliers (SiPM) detectors. SiPMs are seeing an increasingly widespread use in acceleratorbased particle and nuclear physics experiment. Recent developments in SiPM technology made them ideal candidates for the many different applications of scintillation crystals readout, including homeland security, thanks to their large dynamic range, quantum efficiency, robustness and immunity to external magnetic fields.

\section{References}

1. Chen $Y$ et al (2016) Stability of the baseline holder in readout circuits for radiation detectors. IEEE Trans Nucl Sci 63(1):316-324

2. De Geronimo G, O'Connor P, Grosholz J (1999) A CMOS baseline holder (BLH) for readout ASICs. In: 1999 IEEE nuclear science symposium. Conference record. 1999 nuclear science symposium and medical imaging conference (Cat. No. 99CH37019), vol 1. IEEE, pp 370-374

3. Montagnani GL et al (2018) GAMMASiPM ASIC: performance assessment and improved design with $87 \mathrm{~dB}$ dynamic range. In: 2018 IEEE nuclear science symposium and medical imaging conference (NSS/MIC). IEEE [To be published]

4. Montagnani GL et al (2019) Spectroscopic performance of a Sr co-doped 3" LaBr3 scintillator read by a SiPM array. Nucl Instrum Methods Phys Res Sect A: Accel Spectrom Detect Assoc Equip 931:158-161

5. Montagnani GL et al (2019) A compact 4-decade dynamic range readout module for gamma spectroscopy and imaging. In: 2019 IEEE international symposium on circuits and systems (ISCAS). IEEE, pp 1-5

Open Access This chapter is licensed under the terms of the Creative Commons Attribution 4.0 International License (http://creativecommons.org/licenses/by/4.0/), which permits use, sharing, adaptation, distribution and reproduction in any medium or format, as long as you give appropriate credit to the original author(s) and the source, provide a link to the Creative Commons license and indicate if changes were made.

The images or other third party material in this chapter are included in the chapter's Creative Commons license, unless indicated otherwise in a credit line to the material. If material is not included in the chapter's Creative Commons license and your intended use is not permitted by statutory regulation or exceeds the permitted use, you will need to obtain permission directly from the copyright holder.

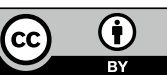

